\title{
POLINEUROPATÍA Y MIOPATÍA EN EL PACIENTE CRÍTICO REVISIÓN DE LA LITERATURA
}

\author{
César Enciso MD*, Ernesto Ojeda MD**, Francy Castro MD***
}

\section{Resumen}

La polineuropatía y la miopatía del paciente crítico son complicaciones frecuentes en la UCI que se caracterizan por debilidad muscular generalizada incluyendo la limitación en el destete de la ventilación mecánica, prolongando la estancia hospitalaria y el riesgo de otras complicaciones. Causan discapacidad crónica en los sobrevivientes de la enfermedad crítica debido a cambios funcionales y estructurales en las fibras nerviosas y musculares resultando en la degeneración del nervio axonal, pérdida de miosina del músculo, hipoxia celular y necrosis muscular, lo que conduce a la inexcitabilidad eléctrica de nervios y músculos, con debilidad muscular reversible. El diagnóstico se realiza mediante la clínica, estudios electrofisiológicos y biopsia muscular. El control metabólico y de factores de riesgo reducirian la gravedad de las complicaciones y la rehabilitación temprana en la unidad podría acelerar la recuperación funcional y la independencia de los pacientes.

Palabras clave: polineuropatía, miopatía, electromiografía, neuroconducción.

Abreviaturas: UCI, unidad de cuidados intensivos; PPC, polineuropatía de paciente crítico; MPC, miopatía del paciente crítico.

\section{POLYNEUROPATHY AND MYOPATHY IN CRITICALLY ILL PATIENTS - A LITERATURE REVIEW}

\section{Abstract}

Critical illness polyneuropathy and myopathy are frequent complications in patients admitted to the ICU distinguished by generalized muscle weakness which hinders weaning from mechanical ventilation thus prolonging length of hospitalization and posing a higher risk of other complications. They cause chronic disability in survivors of critical illness due to functional and structural changes in nervous and muscular fibers resulting in axonal degeneration, loss of myosin filaments, cell hypoxia and muscle necrosis leading to nerve and muscle electrical inexcitability causing reversible muscle weakness. Clinical features and electrophysiologic testing or muscle biopsy are the gold standard for diagnosis. Controlling metabolic factors and risk factors could reduce serious complications and rehabilitation at an early stage of the ICU stay could improve functional recovery and patient independence.

Key words: polyneuropathy, myopathy, electromyography, nerve conduction

Fecha recibido: julio 23 de 2012 - Fecha aceptado: marzo 18 de 2013

* Anestesiólogo, Medicina crítica y cuidado intensivo. Instructor Asociado, Fundación Universitaria de Ciencias de la Salud, Bogotá DC, Colombia.

\footnotetext{
** Neurólogo, neurofisiólogo. Instructor Asistente, Fundación Universitaria de Ciencias de la Salud, Bogotá DC, Colombia.

*** Residente IV de Neurología, Fundación Universitaria de Ciencias de la Salud, Bogotá DC, Colombia.
} 


\section{Introducción}

El desarrollo de nuevas tecnologías y de un proceso unificado de atención, ha tenido un impacto positivo en la supervivencia de pacientes que por la severidad de su enfermedad requieren como parte del manejo soporte en UCI. ${ }^{1,2}$ La mayor supervivencia ante episodios que en épocas anteriores eran siempre letales, genera una población de pacientes con características muy específicas, en la cual aparecen nuevos síndromes como los cuadros de polineuromiopatía no clasificados dentro de otras enfermedades, surgiendo como un factor independiente que tiene impacto en los tiempos de estancia y ventilación mecánica, así como en la infección, discapacidad y mortalidad. ${ }^{3,4}$

La primera descripción de este grupo de patologías aparece hacia 1977, en un paciente asmático que requirió durante su atención la administración de altas dosis de esteroides y de relajantes musculares no despolarizantes. ${ }^{5}$ Después Bolton y col. en $1986^{6}$ la describieron como una alteración neuromuscular definida así por el daño tanto en el nervio periférico como en el músculo o la unión neuromuscular, asociándola con la estancia en la UCI y la aparición de un importante compromiso motor en las extremidades con un curso que puede ser severo y prolongado. ${ }^{2}$ A partir de este momento el interés ha sido creciente al observar la severidad del compromiso y la discapacidad relacionada con el impacto económico que tiene dentro del sistema de salud la atención de estos pacientes, algunos de los cuales nunca logran una rehabilitación suficiente para reincorporarse a una actividad productiva o incluso sin recuperación completa del autocuidado. ${ }^{7}$

Las causas asociadas con este grupo de patologías han sido múltiples y las relaciones no han sido tan claras. Se describe el uso concomitante de relajantes musculares y altas dosis de esteroides, aunque la administración separada de este tipo de medicamentos también se ha observado como factor desencadenante ${ }^{8,9}$, la infección en todos los estadios de clasificación desde la sepsis severa hasta el cuadro de disfunción multiorgánica ${ }^{10,11}$, la diabetes mellitus, la hiperglicemia no controlada, el uso de algunos antibióticos como los aminoglucósidos con reconocido efecto en la unión neuromuscular y otros eventos en los cuales la causalidad no siempre se ha demostrado. ${ }^{12-15}$

El diagnóstico se basa tanto en la clínica como en los estudios neurofisiológicos, que son una herramienta importante en el estudio de diferentes patologías que comprometen el sistema nervioso central y periférico. ${ }^{16}$ Los estudios de electromiografía (EMG) y velocidad de neuroconducción (VNC) que se consideran una extensión del examen neurológico, permiten detectar el compromiso subclínico de los nervios asociados con patologías sistémicas.

Los potenciales viajan a lo largo del nervio estimulado y generan en el músculo un potencial de acción de tipo motor compuesto $(P A M C)$ y otro de acción nervioso sensitivo (PANS). ${ }^{17}$ Este tipo de prueba evalúa la unidad motora (motoneurona alfa, su axón y todas las fibras musculares extrafusales) por medio del registro de los potenciales en la contracción de las fibras musculares que ella inerva y que se reclutan en el esfuerzo voluntario mínimo o máximo. ${ }^{18}$

La EMG permite observar cambios en la morfología del potencial de la unidad motora y los secundarios al compromiso de la fibra muscular, el nervio, la raíz o la motoneurona. ${ }^{17-19} \mathrm{El}$ tiempo de conducción se estudia a través de la latencia (definida como el tiempo que tarda en registrarse el potencial de acción desde que se da el estímulo eléctrico), la dispersión (duración del potencial de acción), bloqueo (cuando en un determinado punto de estimulación disminuye en forma brusca la amplitud del potencial de acción), onda $F$ y reflejo $H$ (reflejos de larga latencia o radiculares; miden no sólo la conducción del nervio periférico sino también el segmento radicular) y la velocidad de conducción (característica de la transmisión nerviosa, oscila entre 50 y $60 \mathrm{mt} / \mathrm{seg}$ ). El tamaño del nervio se estudia mediante la amplitud de la respuesta que refleja el número de axones, los nervios motores se miden en milivoltios y los sensitivos en microvoltios. ${ }^{17}$ En una lesión nerviosa en la que se produce un daño de los axones, la amplitud del potencial de acción se encuentra reducida como es el caso de la PPC. ${ }^{10,17,18,20}$ 
Los desenlaces que han sido estudiados de manera más amplia corresponden al tiempo de ventilación mecánica y al de estancia en $\mathrm{UCI}^{15,21}$ y en una menor proporción la infección, la mortalidad y la discapacidad, sin embargo los estudios en su mayoría se realizan en grupos de pacientes en quienes la sintomatología orientó a la búsqueda activa de la alteración neuromuscular ${ }^{16}$, desconociendo la proporción de aquellos ventilados por un tiempo prolongado que tengan un compromiso neuromuscular no evidenciado por el grupo de soporte en la UCI y las implicaciones que este compromiso pueda desencadenar. ${ }^{10,12,15}$

Por tanto, el enfoque diagnóstico es muy importante. El manejo de la PPC y la MPC se basa en el tratamiento de apoyo, control de glicemia, terapia de sepsis y falla multiorgánica en curso. La evidencia reciente sugiere que el diagnóstico y la rehabilitación temprana efectiva mejora la funcionalidad e independencia y la calidad de vida al egreso de la unidad. ${ }^{13,16,22}$ En esta revisión se describen la incidencia, factores de riesgo, enfoque clínico y electrofisiológico, características histológicas de la PPC y la MPC y los avances en la rehabilitación temprana,

\section{Polineuropatía del paciente crítico}

Características clínicas: la PPC es una complicación importante relacionada con la atención en la UCI. El cuadro de debilidad con predominio en miembros inferiores relacionado con la enfermedad crítica, se ha descrito desde el punto de vista fisiopatológico como una polineuropatía distal sensitivo motora de tipo axonal, de presentación simétrica, con predominio de extremidades inferiores y músculos respiratorios ${ }^{16,23}$ (Tabla 1). La incidencia reportada depende de la población que se analice, del tipo de unidad de los factores de riesgo a los cuales fue expuesta la población, criterios diagnósticos usados y el momento de reconocer la enfermedad aguda. ${ }^{19}$

Algunos estudios sugieren que la polineuropatía puede estar presente incluso desde la primera semana de admisión de los pacientes a la UCI y pasar inadvertida por la administración de fármacos sedantes y un soporte ventilatorio alto, o por alteraciones en el nivel de conciencia que no hacen evidente el grado de compromiso motor. ${ }^{9,24}$

El examen físico en paciente bajo sedación es difícil tanto en la realización como la interpretación, es necesario observar respuestas sutiles como cambios en los signos vitales o gestos relacionados con estímulos de dolor, movilización escasa o nula de extremidades, flacidez y debilidad casi siempre simétrica, disminución o ausencia de los reflejos osteotendinosos profundos y en el paciente alerta además de estos signos y con mayor facilidad disminución o pérdida distal de la sensibilidad al dolor, temperatura y vibración. ${ }^{2,19,24,25}$

En los casos más severos los signos de mayor peso corresponden al compromiso ventilatorio, lo cual se ha relacionado con el del nervio frénico y de los músculos intercostales y el diafragma ${ }^{10,26,27}$, hallazgo muy común y que puede ser un síntoma permanente. La PPC es un factor de riesgo independiente para el destete fallido de la ventilación mecánica y la prolongación de la misma. ${ }^{27}$

Estos hallazgos representan la respuesta del sistema nervioso periférico a los eventos relacionados con la enfermedad crítica; pero también se ha descrito un cuadro de encefalopatía difusa en etapas tempranas de la enfermedad aguda sugiriendo compromiso del sistema nervioso central. ${ }^{24}$

\section{Características electrofisiológicas e histológicas:} la electromiografía y los estudios de conducción nerviosa son útiles para confirmar el diagnóstico,

Tabla I. Criterios diagnósticos de PPC $<+$

I. Paciente en estado crítico(disfunción y/o falla multiorgánica).

2. Debilidad en las extremidadeso dificultad parael destete del ventilador después de excluir causas neuromusculares, pulmonares o cardíacas.

3. Evidencia electrofisiológica de polineuropatía axonal motora y sensitiva.

4. Ausencia o disminución de respuesta al estímulo repetido de los nervios a estudio.

*Diagnóstico definitivo de PPC, cuatro criterios diagnósticos; probable, criterios I,3 y 4; y diagnóstico de debilidad adquirida en la $\mathrm{UCl}$, criterios I y $2 .{ }^{24}$ 
así como para diferenciar la lesión nerviosa de la muscular o determinar el compromiso mixto. Las neuroconducciones muestran disminución en la amplitud del potencial de acción muscular motor compuesto (PAMC) y del potencial de acción nervioso sensitivo (PANS), con velocidad de conducción disminuida o normal. ${ }^{16,17}$

Estas pruebas indican además el grado de compromiso funcional, ya sea leve, moderado o severo, y sugiere un tiempo de evolución agudo, subagudo o crónico. La denervación produce ondas agudas positivas (reflejan irritabilidad de las fibras musculares), potenciales de fibrilación y descargas repetitivas complejas. ${ }^{17}$ Los hallazgos no son específicos ya que estos cambios pueden estar presentes tanto en las dos patologías (PPC-MPC) y en otros trastornos neuromusculares. Por lo tanto la diferenciación debe realizarse mediante patología $a^{28}$, incrementando el valor de los estudios electrofisiológicos, con hallazgos concluyentes cuando los pacientes cooperan y hay registro del reclutamiento voluntario de la unidad del potencial motor. ${ }^{15,17,29}$

Las ondas positivas y el potencial de fibrilación tienden a desaparecer y surgen las ondas polifásicas como signo de reinervación muscular con aumento de la amplitud en los pacientes en quienes ocurre recuperación. ${ }^{17,20,29}$ La biopsia de nervio se reserva cuando es difícil establecer si el proceso es predominante axonal o desmielinizante, o bien si hay síntomas que sugieren compromiso primario de fibra pequeña en las cuales la EMG no es capaz de evaluarlas en forma efectiva.

El nervio que suele emplearse como referencia es el sural al nivel del maléolo; como es sensitivo, no es el ideal en neuropatías motoras, en estos últimos casos se aconseja tomar biopsia del peronero profundo o del radial.

Los estudios mediante biopsia de nervio y músculo confirman los hallazgos electrofisiológicos de una degeneración axonal distal primaria motora y sensitiva, con denervación y atrofia muscular. Evidencia cromatolisis de las células del asta anterior indicando daño axonal. La biopsia muscular en el PPC revela denervación aguda del músculo con atrofia de fibras tipos I y II..$^{16,30}$

\section{Miopatía del paciente crítico}

Es una afección primaria cuya clínica, es similar a la descrita en la PPC con dificultad para retirarlo del ventilador, flacidez de las extremidades, posible disminución en los reflejos osteotendinosos profundos y conservación de la sensibilidad (Tabla 2).,31,32

Características electrofisiológicas e histológicas: los principales hallazgos en el PAMC son la reducción de la amplitud e incremento en la duración. El potencial de acción nervioso sensitivo puede ser normal con disminución en la respuesta con aguja del potencial de unión motor miopático a estimulación directa. La reducción de la excitabilidad de la membrana muscular está establecida por la diferencia entre la amplitud de PAMC después de la estimulación nerviosa y la directa muscular, que está por debajo de 0.5 si esta última es menor $3 \mathrm{mV} \cdot{ }^{18,33,34}$

La duración del PAMC es un signo de miopatía que llega a ser dos o tres veces más larga que en los pacientes sanos, más frecuente en extremidades inferiores ${ }^{35-37}$, con disminución en la amplitud, aunque en las patologías desmielinizantes puede aumentar sobre todo a nivel proximal. En la neuropatía axonal como en la PPC la duración no está aumentada ${ }^{35}$, pero si además hay compromiso del nervio frénico, estos estudios de conducción muestran disminución en la amplitud, sin cambios en la latencia y presencia de fibrilaciones y ondas positivas a la estimulación eléctrica con aguja en los músculos diafragmáticos. ${ }^{6}$

Los hallazgos histopatológicos incluidos en todos los casos evidenciaron atrofia de la fibra muscular, degeneración, regeneración, cambios nucleares y reducción de la actividad miofibrilar en fibras tipo I y II. ${ }^{38} \mathrm{Se}$ han realizado diversas clasificaciones microscópicas. ${ }^{39}$ Hunt propuso la de miopatías del paciente crítico, de filamento grueso y la necrotizante basada en los cambios existentes. . $^{74,37}$ 
La presentación mixta PPC y MPC va de leve a severa y la manifestación común es la debilidad de las extremidades. ${ }^{9,33}$ En la forma leve la duración del PAMC está reducida y la electromiografía muestra ondas lentas y escasa fibrilación, ondas positivas. ${ }^{32}$ La biopsia por lo regular es normal y la recuperación buena. ${ }^{12} \mathrm{En}$ la severa hay disminución de la amplitud del PAMC y el PANS está ausente, si la latencia es normal o tiene mínima afectación sugiere degeneración axonal de la fibra nerviosa sensitiva. ${ }^{20}$

La biopsia suele mostrar necrosis. En casos reportados con controles a corto y mediano plazo informan la discapacidad persistente por debilidad muscular y como hallazgo describen que no hay verdadera correlación entre el diagnóstico clínico y el electrofisiológico. El seguimiento mostró que aquellos con solo miopatía tenían mejor recuperación mientras que con PPC moderada a severa requerían rehabilitación prolongada y permanente. ${ }^{40,41}$

\section{Incidencia, factores de riesgo y fisiopatología}

A pesar de las limitaciones los estudios prospectivos utilizando estas pruebas neurofisiológicas revelan que la polineuromiopatía se encuentra presente en 33 a $57 \%$ de los pacientes que permanecen en la UCI por más de siete días ${ }^{26,27}$ y que es aún mayor (68-100\%) cuando hay presencia de sepsis o síndrome de respuesta inflamatoria sistémica (SIRS). ${ }^{7,15,42}$

En estas dos situaciones mencionadas la frecuencia puede ser tan alta como $70 \%^{10}$, con posibilidad de llegar al $100 \%$ cuando aparece falla multiorgánica como complicación. ${ }^{43}$ Otros pacientes con alto riesgo son quienes tienen como diagnóstico de ingreso o que desarrollan durante su estancia en UCI un cuadro de SDRA, con una frecuencia del $60 \%{ }^{27,44}$ relacionada con la necesidad de relajación muscular y de soporte ventilatorio mecánico prolongado, que aparece como una variable independiente, observando que en pacientes con un tiempo de ventilación mecánica superior a siete días ocurre entre 25 y $30 \%$, ascendiendo a $58 \%$ cuando la herramienta de búsqueda es una prueba neurofisiológica. ${ }^{26,45,46}$

La fisiopatología de estas entidades es poco clara y compleja; el daño en la microcirculación se ha postulado como mecanismo causal en el nervio periférico resaltando el papel de las moléculas inflamatorias, vasodilatación excesiva y la agregación de elementos celulares a través de la activación de moléculas de adhesión. ${ }^{47}$

Hace poco se demostró el aumento de expresión de E-selectina (considerada como marcador de actividad celular) en el endotelio epineurial y endoneural de los vasos de los nervios periféricos en pacientes con

\section{Tabla 2. Características clínicas de la MPC*}

I. Paciente con enfermedad crítica (falla multiorgánica).

2. Dificultad para el destete de la ventilación mecánica después de descartar enfermedad cardíaca y pulmonar.

3. Amplitud del PAMC menor del $80 \%$ del límite normal bajo en dos o más nervios sin bloqueos de conducción.

4. Amplitud del potencial de acción motor sensitivo PANS menor del $80 \%$ del límite inferior normal.

5. El potencial de la unidad motora con electromiografía de aguja es de corta duración, baja amplitud, con o sin potenciales de fibrilación y contracción completa. Si el paciente está alerta, hay incremento de duración PAMC o reducción de excitabilidad de la membrana muscular a la estimulación directa en el paciente bajo sedación.

6. Ausencia o disminución de la respuesta a la estimulación nerviosa repetitiva.

7. Hallazgos histológicos de miopatía primaria (pérdida de miosina o necrosis muscular).

* Diagnóstico definitivo: 7 criterios; diagnóstico probable I y 3-6 criterios; y diagnóstico de debilidad adquirida en UCl solo criterios I y 2 . 
estado crítico séptico. ${ }^{43} \mathrm{Su}$ expresión se incrementa con la inducción de citoquinas proinflamatorias como el TNF- $\alpha$ y IL1, promoviendo la adhesión endotelio-leucocito y extravasación de leucocitos activados entre el espacio endoneural, esto lleva a lesión titular con producción de citoquinas locales y aumenta la permeabilidad vascular favoreciendo el paso de los factores neurotóxicos al endoneuro. ${ }^{44,46}$ Esto explica la mayor frecuencia del cuadro en pacientes con sepsis y en general en los pacientes críticos, al relacionar la enfermedad crítica con una condición compleja en la que eventos metabólicos e inflamatorios a nivel vascular y celular coexisten como parte de la fisiopatología de los diferentes procesos..$^{23,31}$

En los últimos años se han hecho importantes avances en la búsqueda de una explicación, de cómo los músculos pueden ser inexcitables a pesar de mantener una estructura normal. Se encontró denervación inducida por la reducción del potencial en reposo del músculo (valor normal $-85 \mathrm{mV}$ a $-60 \mathrm{mV}$ ), la falta de regulación de conducción de cloro en la membrana posdenervación y la inactivación rápida de los canales de sodio voltaje dependientes que los hace llegar a potenciales más negativos $(-11 \mathrm{mV}) .{ }^{48,49} \mathrm{El}$ cambio de hiperpolarización que ocasiona la inactivación de canales rápidos de sodio dependientes de voltaje es de crucial importancia en la reducción de la excitabilidad de fibras musculares denervadas y casi siempre se debe, aunque no es exclusivo, a esteroides ${ }^{44,49,50} \mathrm{La}$ disfunción mitocondrial es otro mecanismo importante en la fisiopatología de la miopatía por depleción de ATP y de glutatión intracelular, así como producción de óxido nítrico en el músculo esquelético, cambiando la proporción de un óxido nítrico constitutivo a inducible que no se diferencia del fenómeno global de los radicales libres de oxígeno en la enfermedad crítica. $^{51}$

El daño o la inhibición de las subunidades 1 del complejo mitocondrial en la cadena de trasporte de electrones es responsable de la disminución de la capacidad para generar ATP. Este fallo bioenergético es por tanto un importante mecanismo patogénico subyacente inducido por el fenómeno séptico y que lleva a daño muscular, similar al descrito en la lesión de nervio periférico. El efecto final del daño en la fibra muscular está dado por la alteración en el sistema proteolítico intracelular, es decir, compromiso de la ubiquitina-proteasoma y calpaínas, sistema lisosomal y no lisosomal..$^{52,53}$ Las citoquinas proinflamatorias, así como la disminución de los niveles de hormonas anabólicas (insulina e insulina-like factor de crecimiento-1) y el incremento de los niveles de hormonas catabólicas (cortisol, catecolaminas y glucagón), generan un fuerte efecto catabólico dejando disponibles aminoácidos musculares claves en este proceso. ${ }^{19,24,31}$

La activación de la vía ubiquitine-ligasa es común en las atrofias neurogénica y miogénica, solo que en los pacientes con MPP la activación se genera por la vía factor de crecimiento (TGF) -beta /MAPK. ${ }^{52,53}$

Los aminoglucósidos han recibido particular atención en dos estudios asociados con su uso y PPC. ${ }^{11,46} \mathrm{Sin}$ embargo, otros autores no pudieron confirmar estos resultados ${ }^{10,24,26,54,55}$ y parece que estas asociaciones no siempre son causales de la patología. Se han identificado factores de riesgo en estudios prospectivos: sexo femenino ${ }^{12}$, duración de la disfunción orgánica, insuficiencia renal y terapia de reemplazo renal, hiperosmolaridad, gravedad ${ }^{9,56}$ de la enfermedad, hipoalbuminemia ${ }^{10}$, nutrición parenteral ${ }^{13,24}$, tiempo de estancia en UCI e hiperglicemia. ${ }^{56}$

El impacto de los corticosteroides en la función neuromuscular en la UCI ha sido objeto de controversia. Muchos informes han encontrado que el MPC ocurre en los pacientes tratados con una combinación de corticoesteroides y los bloqueantes neuromusculares (BNM), siendo la miopatía más frecuente en asociación con enfermedad pulmonar obstructiva crónica y asma grave..$^{28,57}$ Algunos estudios no identificaron BNM y corticoides como factor de riesgo independiente para el PPC o PPC/MPC ${ }^{58}$, aunque otros dos si lo hallaron. ${ }^{8,20} \mathrm{La} \mathrm{MPC}$ ha sido reportada en pacientes que recibieron sólo uno de estos agentes o ninguno, por tanto estos fármacos no son esenciales en el desarrollo de PPC, MPP o ambas. ${ }^{28,39,59}$ La elevación de la CK sérica se empleó como marcador de daño muscular, encontrándose en algunos grupos dentro de un valor normal o con moderada elevación. Su significado 
orienta hacia el diagnóstico de miopatías necrosantes, con potencial de acción de unidad motora disminuido en amplitud y duración, polifásico, con reclutamiento precoz y mortalidad superior a $70 \% .{ }^{29}$

\section{Diagnóstico diferencial y final}

La PPC y MPP tienen un importante impacto en la evolución del paciente en la UCI aun después de su egreso, por tanto requiere un diagnóstico temprano. El retiro de la sedación en la UCI permite observar la debilidad y la flacidez de las extremidades ${ }^{22}$, pues el que permanece en coma o bajo sedación retira las extremidades en forma leve ante el estímulo doloroso fuerte, con gesto facial de dolor y los reflejos osteotendinosos pueden estar preservados o disminuidos. La dificultad en el retiro en la ventilación mecánica es un signo importante de enfermedad. Debe sospecharse cuando la debilidad no mejora o si la realización de la extubación es fallida después de corregir otras etiologías como posibles causas ${ }^{22}$ (Tablas 1 y 2).

Los estudios electrofisiológicos y de biopsia muscular confirman la presencia de PPC o MPC o ambas, pero no siempre se interrelacionan. ${ }^{60}$ Se requiere correlación entre los resultados neurofisiológicos y de la biopsia, ya que la mayor severidad se evidencia con la prueba histopatológica aun con demostración de enfermedad leve tanto en la electromiografía como en la neuroconducción. Sin embargo existen resultados contradictorios debido al pequeño número de estudios prospectivos que combinan estas técnicas. ${ }^{15,59}$ La MPC y PPC son complicaciones que surgen de la enfermedad crítica, las cuales pueden aparecer desde los primeros días del ingreso a la unidad o del requerimiento de ventilación mecánica invasiva. ${ }^{7,15,21} \mathrm{La}$ debilidad y la paresia son signos de otras patologías neurológicas que involucran cerebro, medula espinal, nervios periféricos, unión neuromuscular y de los propios músculos. La alteración hidroelectrolítica como la hipocalemia y la hipofosfatemia pueden causar miopatía aguda y la hipermagnesemia puede alterar la trasmisión neuromuscular. La sepsis por si sola no es causa de alteración en la trasmisión neuromuscular, pero el uso de agentes vasopresores, algunos antimicrobianos y el empleo concomitante de medicamentos que generan neurotoxicidad como antirretrovirales, quimioterápicos e infusión de hipnóticos como el propofol, pueden desencadenar el cuadro. ${ }^{61}$ El diagnóstico diferencial más importante de PPC es el síndrome de Guillain Barre con cuadro de debilidad progresiva simétrica, arrefléxica, que puede aparecer dos a cuatro semanas después del antecedente de diarrea e infección por Campylobacter jejuni. En esta patología puede comprometerse la musculatura facial, que no se afecta en la PPC, y la progresión a falla ventilatoria es rápida. Los hallazgos electrodiagnósticos evidencian lesiones principalmente desmielinizantes; el diagnóstico diferencial del PPC es con la variante axonal sensitivo motora ${ }^{62-64}$, con presencia de disociación albumino citológica $^{64}$, en pacientes politraumatizados sometidos a múltiples intervenciones quirúrgicas que desarrollan neuropatía axonal inflamatoria sin lesión por estiramiento ni compresiva. ${ }^{65}$

\section{Tratamiento}

El tratamiento está enfocado hacia la prevención, disminuyendo la exposición a los factores de riesgo descritos; no hay un manejo especifico, se han descrito terapias con inmunoglobulina, hormonas, antioxidantes y control metabólico y nutricional, no tanto para la disminución de la incidencia de presentación de la enfermedad sino el grado de severidad de PPC y MPC. ${ }^{56,66} \mathrm{El}$ empleo de insulina en la terapia intensiva con concentraciones entre 4 y $6 \mathrm{mmol} / \mathrm{l}$, reduce la incidencia tanto en el diagnóstico clínico como en el electrofisiológico, sin embargo es amplia la evidencia en contra de un control estricto que favorece la aparición de hipoglicemia y no hay estudios concluyentes que permitan observar la disminución en los resultados histopatológicos, tanto en pacientes con ingreso clínico como quirúrgico. ${ }^{10,55,56}$ Los niveles óptimos no han sido del todo determinados, pues la terapia intensiva hacia la normoglicemia ha mostrado incremento en la mortalidad del paciente crítico ${ }^{67}$ Otro enfoque hacia el tratamiento es la rehabilitación temprana desde la sospecha clínica de la PPC o MPC, lo que ha permitido la disminución en los días de estancia hospitalaria y mejor pronóstico en cuanto a calidad de vida e independencia funcional en los pacientes que egresan de la UCI. ${ }^{68,69}$ 


\section{Conclusiones}

La PPC y/o MPC son complicaciones comunes como causa de debilidad muscular, parálisis con limitación en la rehabilitación hasta en el $100 \%$ de los casos de pacientes que requieren estancia en la unidad de más de una semana y aumenta en forma proporcional si el requerimiento es mayor. Estas patologías por si mismas prolongan la necesidad de soporte ventilatorio y como resultado limitación en la independencia en actividades de la vida diaria. A pesar de los avances en la descripción de factores de riesgo asociados, continúan siendo patologías subdiagnosticadas; en primera instancia porque se le da prioridad a la enfermedad que motiva el ingreso a la UCI y en segundo lugar aparecen otros factores más comunes como causa de debilidad sobre todo en la dificultad en el destete del ventilador, por ejemplo afectación estructural pulmonar o cardíaca y no por compromiso de músculos diafragmáticos por neuropatía del nervio frénico, lo que lleva al paciente a mayor riesgo de complicaciones; si bien este dato podría explicarse por la mayor gravedad al ingreso más que por la contribución específica de la complicación neurológica. La evidencia muestra que su comportamiento es el de un factor independiente de mortalidad relacionada con complicaciones secundarias tales como neumonía, trombosis venosa profunda y embolismo pulmonar.

Además, PPC y/o MPC se asocian como factor importante a la readmisión en la UCI, secundaria a la debilidad de los músculos respiratorios después del alta, en los pacientes que fueron destetados con éxito. Por último se describe la recuperación, la cual se caracteriza por la reinervación progresiva de los músculos y restauración de la sensibilidad, siendo completa en la mayoría de los casos, con neuropatía leve a moderada a los tres meses; en cambio los sobrevivientes a una sepsis severa con prolongada estancia en UCI tienen recuperación incompleta o puede no ocurrir cuando se encuentra como predictor de discapacidad funcional prolongada un potencial de acción motor compuesto (CMAP) ausente o disminuido en estudios neurofisiológicos. Estas patologías conllevan una gran morbilidad, retrasa el alta de la UCI, la hospitalaria y una vez en su domicilio la calidad de vida es inferior a la de los enfermos que no desarrollan este síndrome.
Hasta donde sabemos no se han publicado datos en nuestro medio acerca de esta patología, que consideramos importante por el impacto descrito en la supervivencia y la calidad de vida del paciente crítico, aún después del egreso de la unidad. No hay resultados de la incidencia de polineuromiopatía identificados mediante examen físico inicial comparado con hallazgos electromiográficos y estudio histopatológico en nuestra población que requiere cuidado crítico; por lo que se hace relevante para el clínico conocer dichos resultados con el fin de realizar el diagnóstico rápido y así mismo plantear intervenciones terapéuticas con un enfoque más temprano que después pueda demostrar efectos en la calidad de vida futura de los pacientes que requieran hospitalización en la unidad.

\section{Referencias}

1. Johnson KL. Neuromuscular complications in the intensive care unit: critical illness polyneuromyopathy. AACN Adv Crit Care. 2007;18(2):167-80; quiz 81-2.

2. Lacomis D, Zochodne DW, Bird SJ. Critical illness myopathy. Muscle Nerve. 2000;23(12):1785-8

3. Leijten FS, de Weerd AW. Critical illness polyneuropathy. A review of the literature, definition and pathophysiology. Clin Neurol Neurosurg. 1994;96(1):10-9.

4. Berek K, Margreiter J, Willeit J, Berek A, Schmutzhard E, Mutz NJ. Polyneuropathies in critically ill patients: a prospective evaluation. Intensive Care Med. 1996;22(9):849-55.

5. MacFarlane IA, Rosenthal FD. Severe myopathy after status asthmaticus. Lancet. 1977;2(8038):615

6. Bolton CF, Laverty DA, Brown JD, Witt NJ, Hahn AF, Sibbald WJ. Critically ill polyneuropathy: electrophysiological studies and differentiation from GuillainBarre syndrome. J Neurol Neurosurg Psychiatry. 1986;49(5):563-73.

7. Kerbaul F, Brousse M, Collart F, Pellissier JF, Planche D, Fernandez C, et al Combination of histopathological and electromyographic patterns can help to evaluate functional outcome of critical ill patients with neuromuscular weakness syndromes. Crit Care. 2004;8(6):R358-66.

8. Geller TJ, Kaiboriboon K, Fenton GA, Hayat GR. Vecuronium-associated axonal motor neuropathy: a variant of critical illness polyneuropathy? Neuromuscul Disord. 2001;11(6-7):579-82.

9. Bednarik J, Vondracek P, Dusek L, Moravcova E, Cundrle I. Risk factors for critical illness polyneuromyopathy. J Neurol. 2005;252(3):343-51.

10. Witt NJ, Zochodne DW, Bolton CF, Grand'Maison F, Wells G, Young GB, et al. Peripheral nerve function in sepsis and multiple organ failure. Chest 1991;99(1):176-84.

11. Leijten FS, De Weerd AW, Poortvliet DC, De Ridder VA, Ulrich C, Harink-De Weerd JE. Critical illness polyneuropathy in multiple organ dysfunction syndrome and weaning from the ventilator. Intensive Care Med. 1996;22(9):856-61.

12. De Jonghe B, Cook D, Sharshar T, Lefaucheur JP, Carlet J, Outin H. Acquired neuromuscular disorders in critically ill patients: a systematic review. Groupe de Reflexion et d'Etude sur les Neuromyopathies En Reanimation. Intensive Care Med. 1998;24(12):1242-50.

13. Marino PL, Millili JJ. Possible role of dietary lipids in critical illness polyneuropathy. Intensive Care Med. 1998;24(1):87. 
14. Schefold JC, Bierbrauer J, Weber-Carstens S. Intensive care unit-acquired weakness (ICUAW) and muscle wasting in critically ill patients with severe sepsis and septic shock. J Cachex Sarcopenia Muscle. 2010;1(2):147-57.

15. Coakley JH, Nagendran K, Honavar M, Hinds CJ. Preliminary observations on the neuromuscular abnormalities in patients with organ failure and sepsis. Intensive Care Med. 1993;19(6):323-8

16. Zifko UA, Zipko HT, Bolton CF. Clinical and electrophysiological findings in critical illness polyneuropathy. J Neurol Sci. 1998;159(2):186-93.

17. Oh SJ. Clinical electromyography nerve conduction studies. 3rd ed. Philadelphia: Lippincott Williams andWilkins; 2003.

18. Trojaborg W, Weimer LH, Hays AP. Electrophysiologic studies in critical illness associated weakness: myopathy or neuropathy - a reappraisal. Clin Neurophysiol. 2001 Sep;112(9):1586-93.

19. Stevens RD, Dowdy DW, Michaels RK, Mendez-Tellez PA, Pronovost PJ, Needham DM. Neuromuscular dysfunction acquired in critical illness: a systematic review. Intensive Care Med. 2007;33(11):1876-91.

20. Visser LH. Critical illness polyneuropathy and myopathy: clinical features, risk factors and prognosis. Eur J Neurol. 2006;13(11):1203-12.

21. De Jonghe B, Sharshar T, Hopkinson N, Outin H. Paresis following mechanical ventilation. Curr Opin Crit Care. 2004;10(1):47-52.

22. De Jonghe B, Bastuji-Garin S, Durand MC, Malissin I, Rodrigues P, Cerf C, et al. Respiratory weakness is associated with limb weakness and delayed weaning in critical illness. Crit Care Med. 2007;35(9):2007-15.

23. Latronico N, Fenzi F, Recupero D, Guarneri B, Tomelleri G, Tonin P, et al. Critical illness myopathy and neuropathy. Lancet. 1996;347(9015):1579-82.

24. Garnacho-Montero J, Madrazo-Osuna J, Garcia-Garmendia JL, Ortiz-Leyba C, Jimenez-Jimenez FJ, Barrero-Almodovar A, et al. Critical illness polyneuropathy: risk factors and clinical consequences. A cohort study in septic patients. Intensive Care Med. 2001;27(8):1288-96.

25. Pandit L, Agrawal A. Neuromuscular disorders in critical illness. Clin Neurol Neurosurg. 2006;108(7):621-7.

26. Bercker S, Weber-Carstens S, Deja M, Grimm C, Wolf S, Behse F, et al. Critical illness polyneuropathy and myopathy in patients with acute respiratory distress syndrome. Crit Care Med. 2005;33(4):711-5.

27. De Jonghe B, Bastuji-Garin S, Sharshar T, Outin H, Brochard L. Does ICUacquired paresis lengthen weaning from mechanical ventilation? Intensive Care Med. 2004;30(6):1117-21.

28. Fernandez-Lorente J, Esteban A, Salinero E, Traba A, Prieto J, Palencia E. [Critical illness myopathy. Neurophysiological and muscular biopsy assessment in 33 patients]. Rev Neurol. 2010;50(12):718-26.

29. Fletcher SN, Kennedy DD, Ghosh IR, Misra VP, Kiff K, Coakley JH, et al. Persistent neuromuscular and neurophysiologic abnormalities in long-term survivors of prolonged critical illness. Crit Care Med. 2003;31(4):1012-6.

30. Zochodne DW, Bolton CF, Wells GA, Gilbert JJ, Hahn AF, Brown JD, et al Critical illness polyneuropathy. A complication of sepsis and multiple organ failure. Brain. 1987;110 ( Pt 4):819-41.

31. Tennila A, Salmi T, Pettila V, Roine RO, Varpula T, Takkunen O. Early signs of critical illness polyneuropathy in ICU patients with systemic inflammatory response syndrome or sepsis. Intensive Care Med. 2000;26(9):1360-3.

32. Bolton CF. Neuromuscular manifestations of critical illness. Muscle Nerve. 2005;32(2):140-63.

33. Lefaucheur JP, Nordine T, Rodriguez P, Brochard L. Origin of ICU acquired paresis determined by direct muscle stimulation. J Neurol Neurosurg Psychiatry. 2006;77(4):500-6.

34. Koch S, Spuler S, Deja M, Bierbrauer J, Dimroth A, Behse F, et al. Critical illness myopathy is frequent: accompanying neuropathy protracts ICU discharge. J Neurol Neurosurg Psychiatry. 2011;82(3):287-93

35. Goodman BP, Harper CM, Boon AJ. Prolonged compound muscle action potential duration in critical illness myopathy. Muscle Nerve. 2009;40(6):1040-2.
36. Ali NA, O'Brien JM, Jr., Hoffmann SP, Phillips G, Garland A, Finley JC, et al. Acquired weakness, handgrip strength, and mortality in critically ill patients. Am J Respir Crit Care Med. 2008;178(3):261-8.

37. Allen DC, Arunachalam R, Mills KR. Critical illness myopathy: further evidence from muscle-fiber excitability studies of an acquired channelopathy. Muscle Nerve. 2008;37(1):14-22.

38. Cohen M. Introduction to peripheral nerves. In: Prayson. R, Goldblum J. Neuropathology. Philadelphia, Pensilvalnia: Elsevier; 2005. p. 565-81

39. Hund E. Myopathy in critically ill patients. Crit Care Med. 1999;27(11):2544-7.

40. Herridge MS, Cheung AM, Tansey CM, Matte-Martyn A, Diaz-Granados N, AlSaidi F, et al. One-year outcomes in survivors of the acute respiratory distress syndrome. N Engl J Med. 2003;348(8):683-93.

41. Guarneri B, Bertolini G, Latronico N. Long-term outcome in patients with critical illness myopathy or neuropathy: the Italian multicentre CRIMYNE study. J Neurol Neurosurg Psychiatry. 2008;79(7):838-41

42. Bolton CF. Sepsis and the systemic inflammatory response syndrome: neuromuscular manifestations. Crit Care Med. 1996;24(8):1408-16.

43. Fenzi F, Latronico N, Refatti N, Rizzuto N. Enhanced expression of E-selectin on the vascular endothelium of peripheral nerve in critically ill patients with neuromuscular disorders. Acta Neuropathol. 2003;106(1):75-82.

44. Druschky A, Herkert M, Radespiel-Troger M, Druschky K, Hund E, Becker $\mathrm{CM}$, et al. Critical illness polyneuropathy: clinical findings and cell culture assay of neurotoxicity assessed by a prospective study. Intensive Care Med. 2001;27(4):686-93

45. Latronico N. Neuromuscular alterations in the critically ill patient: critical illness myopathy, critical illness neuropathy, or both? Intensive Care Med. 2003;29(9):1411-3

46. Fink MP, Evans TW. Mechanisms of organ dysfunction in critical illness: report from a Round Table Conference held in Brussels. Intensive Care Med. 2002;28(3):369-75.

47. Leijten FS, Harinck-de Weerd JE, Poortvliet DC, de Weerd AW. The role of polyneuropathy in motor convalescence after prolonged mechanical ventilation. JAMA. 1995;274(15):1221-5.

48. Rich MM, Pinter MJ, Kraner SD, Barchi RL. Loss of electrical excitability in an animal model of acute quadriplegic myopathy. Ann Neurol. 1998;43(2):171-9.

49. Rich MM, Pinter MJ. Crucial role of sodium channel fast inactivation in muscle fibre inexcitability in a rat model of critical illness myopathy. J Physiol. 2003;547(Pt 2):555-66.

50. Filatov GN, Rich MM. Hyperpolarized shifts in the voltage dependence of fast inactivation of Nav1.4 and Nav1.5 in a rat model of critical illness myopathy. J Physiol. 2004;559(Pt 3):813-20.

51. Brealey D, Brand M, Hargreaves I, Heales S, Land J, Smolenski R, et al. Association between mitochondrial dysfunction and severity and outcome of septic shock. Lancet. 2002;360(9328):219-23.

52. Mitch WE, Goldberg AL. Mechanisms of muscle wasting. The role of the ubiquitin-proteasome pathway. N Engl J Med. 1996;335(25):1897-905.

53. Tiao G, Hobler S, Wang JJ, Meyer TA, Luchette FA, Fischer JE, et al. Sepsis is associated with increased mRNAs of the ubiquitin-proteasome proteolytic pathway in human skeletal muscle. J Clin Invest. 1997;99(2):163-8.

54. Bolton CF, Young GB, Zochodne DW. The neurological complications of sepsis. Ann Neurol. 1993;33(1):94-100. Epub 1993/01/01.

55. Hermans G, De Jonghe B, Bruyninckx F, Van den Berghe G. Interventions for preventing critical illness polyneuropathy and critical illness myopathy. Cochrane Database Syst Rev. 2009(1)

56. Van den Berghe G, Schoonheydt K, Becx P, Bruyninckx F, Wouters PJ. Insulin therapy protects the central and peripheral nervous system of intensive care patients. Neurology. 2005;64(8):1348-53

57. Latronico N, Shehu I, Guarneri B. Use of electrophysiologic testing. Crit Care Med. 2009;37(10 Suppl):S316-20. 
58. de Letter MA, Schmitz PI, Visser LH, Verheul FA, Schellens RL, Op de Coul DA, et al. Risk factors for the development of polyneuropathy and myopathy in critically ill patients. Crit Care Med. 2001;29(12):2281-6.

59. Ahlbeck K, Fredriksson K, Rooyackers O, Maback G, Remahl S, Ansved T, et al. Signs of critical illness polyneuropathy and myopathy can be seen early in the ICU course. Acta Anaesthesiol Scand. 2009;53(6):717-23.

60. Op de Coul AA, Verheul GA, Leyten AC, Schellens RL, Teepen JL. Critical illness polyneuromyopathy after artificial respiration. Clin Neurol Neurosurg. 1991;93(1):27-33.

61. Cremer OL, Moons KG, Bouman EA, Kruijswijk JE, de Smet AM, Kalkman CJ. Long-term propofol infusion and cardiac failure in adult head-injured patients. Lancet. 2001;357(9250):117-8.

62. Bolton CF. The changing concepts of Guillain-Barre syndrome. N Engl J Med. 1995;333(21):1415-7.

63. Hughes RA, Wijdicks EF, Benson E, Cornblath DR, Hahn AF, Meythaler JM, et al. Supportive care for patients with Guillain-Barre syndrome. Arch Neurol 2005;62(8):1194-8.
64. Uncini A, Manzoli C, Notturno F, Capasso M. Pitfalls in electrodiagnosis of Guillain-Barre syndrome subtypes. J Neurol Neurosurg Psychiatry. 2010;81(10):1157-63.

65. Staff NP, Engelstad J, Klein CJ, Amrami KK, Spinner RJ, Dyck PJ, et al. Postsurgical inflammatory neuropathy. Brain. 2010;133(10):2866-80.

66. Mohr M, Englisch L, Roth A, Burchardi H, Zielmann S. Effects of early treatment with immunoglobulin on critical illness polyneuropathy following multiple organ failure and gram-negative sepsis. Intensive Care Med. 1997;23(11):1144-9.

67. NICE-SUGAR Study Investigators, Finfer S, Chittock DR, Su SY, Blair D, Foster $\mathrm{D}$, et al. Intensive versus conventional glucose control in critically ill patients. N Engl J Med. 2009;360(13):1283-97.

68. Schweickert WD, Pohlman MC, Pohlman AS, Nigos C, Pawlik AJ, Esbrook CL, et al. Early physical and occupational therapy in mechanically ventilated, critically ill patients: a randomised controlled trial. Lancet. 2009;373(9678):1874-82.

69. de Seze M, Petit H, Wiart L, Cardinaud JP, Gaujard E, Joseph PA, et al. Critical illness polyneuropathy. A 2-year follow-up study in 19 severe cases. Eur Neurol. 2000;43(2):61-9. 